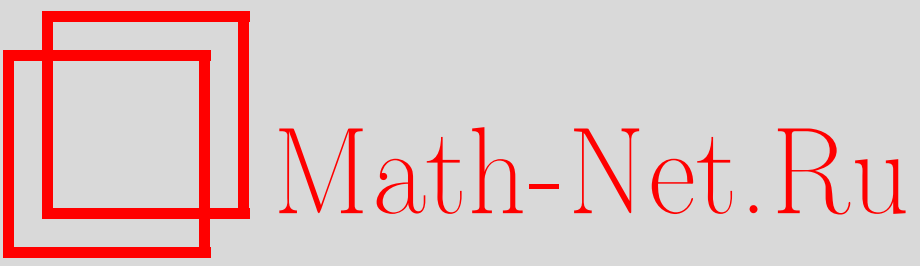

Е. П. Величева, А. А. Сузько, Точные решения нестационарного уравнения Шредингера, ТМФ, 1998, том 115, номер 3, 410-418

DOI: https://doi.org/10.4213/tmf883

Использование Общероссийского математического портала Math-Net.Ru подразумевает, что вы прочитали и согласны с пользовательским соглашением

http://www . mathnet.ru/rus/agreement

Параметры загрузки:

IP: 18.234 .156 .22

26 апреля 2023 г., 13:56:57 
ТЕОРЕТИЧЕСКАЯ

И МАТЕМАТИЧЕСКАЯ

ФИЗИКА

Том 115, № 3

июнь, 1998

(C) $\mathbf{1 9 9 8}$ г.

Е.П. Величева*, А.А. Сузько

\section{ТОЧНЫЕ РЕШЕНИЯ НЕСТАЦИОНАРНОГО УРАВНЕНИЯ ШРЕДИНГЕРА}

Разрабатывается процедура решения в явном аналитическом виде нестационарного уравнения Шредингера на основе точно решаемых стационарных задач. Рассмотрено образование неадиабатической геометрической фазы при циклической эволюции квантовой системы.

\section{1. ВВЕДЕНИЕ}

Предположим, что состояние $|\Psi(t)\rangle$ системы эволюционирует в соответствии с уравнением Шредингера

$$
i \hbar \frac{d|\Psi(t)\rangle}{d t}=H(t)|\Psi(t)\rangle .
$$

Решение этого уравнения с помошью оператора эволюции $U(t)=U(t, 0)$ можно представить в виде

$$
|\Psi(t)\rangle=U(t)|\Psi(0)\rangle .
$$

Для оператора эволюции получаем уравнение

$$
i \hbar \frac{d U(t)}{d t}=H(t) U(t)
$$

решение которого обычно записывают в символической форме

$$
U(t)=T \exp \left[\left(-\frac{i}{\hbar}\right) \int_{0}^{t} H\left(t^{\prime}\right) d t^{\prime}\right]
$$

где $T$ - хронологический оператор. Решение нестационарного уравнения (1) можно также представить с помощью общего унитарного преобразования $\widehat{\mathcal{S}}^{\dagger}(t)=\widehat{\mathcal{S}}^{-1}(t)$ :

$$
|\Psi(t)\rangle=\widehat{\mathcal{S}}(t)|\widehat{\psi}(t)\rangle
$$

\footnotetext{
* Гомельский государственный университет, Гомель, Беларусь

${ }^{\dagger}$ Институт радиационных физико-химических проблем Академии наук Беларуси, Минск, Беларусь
} 
Из того что это преобразование унитарно и сохраняет вид уравнения (1), следуют формулы преобразования исходных гамильтониана и оператора эволюции

$$
\begin{aligned}
H(t) \rightarrow \widehat{H}(t) & =\widehat{\mathcal{S}}^{\dagger}(t) H(t) \widehat{\mathcal{S}}(t)-i \hbar \widehat{\mathcal{S}}^{\dagger}(t) \frac{d}{d t} \widehat{\mathcal{S}}(t), \\
U(t) & \rightarrow \widehat{U}(t)=\widehat{\mathcal{S}}^{\dagger}(t) U(t) \widehat{\mathcal{S}}(0)
\end{aligned}
$$

Легко видеть, что квантовые унитарные преобразования $\widehat{\mathcal{S}}(t)$ действуют аналогично неабелевым калибровочным преобразованиям физики частиц. Чем же могут быть полезны эти квантовые калибровочные преобразования для решения уравнения (1)? Удобно ввести такое калибровочное преобразование $\widehat{\mathcal{S}}(t)=\mathcal{S}(t)$, чтобы в полученном из (1) с его помощью уравнении

$$
i \hbar \frac{d|\Phi(t)\rangle}{d t}=\bar{H}|\Phi(t)\rangle
$$

гамильтониан $\bar{H}(t)$, определяемый соотношением (4), не зависел от времени, т.е. $(d / d t) \bar{H}=0$ (см., например, $[1,2])$. Отсюда следует уравнение для оператора $\mathcal{S}(t)$

$$
\dot{\mathcal{S}}^{\dagger} H \mathcal{S}+\mathcal{S}^{\dagger} \frac{\partial H}{\partial t} \mathcal{S}+\mathcal{S}^{\dagger} H \dot{\mathcal{S}}-i \hbar \dot{\mathcal{S}}^{\dagger} \dot{\mathcal{S}}-i \hbar \mathcal{S}^{\dagger} \ddot{\mathcal{S}}=0
$$

где принято обозначение $\dot{\mathcal{S}}=(d / d t) \mathcal{S}$.

Решение уравнения (6) с не зависяшим от времени гамильтонианом

$$
|\Phi(t)\rangle=\widehat{U}(t, 0)|\Phi(0)\rangle \equiv \exp (-i \bar{H} t / \hbar)|\Phi(0)\rangle
$$

может быть записано в виде

$$
|\Phi(t)\rangle=\exp \left(-\frac{i \mathcal{E} t}{\hbar}\right)|\varphi(\mathcal{E})\rangle
$$

и, по сути дела, сводится к решению стационарной задачи

$$
\bar{H}|\varphi(\mathcal{E})\rangle=\mathcal{E}|\varphi(\mathcal{E})\rangle,
$$

т.е. функции $|\varphi(\mathcal{E})\rangle=|\Phi(t=0)\rangle$ являются собственными функциями оператора $\bar{H}$ и в то же время функциями начального состояния для уравнения (6). Как легко видеть из (8), учет оператора эволюции

$$
\widehat{U}(t)=\exp \left(-\frac{i}{\hbar} \bar{H} t\right)
$$

для $\Phi(t)$ в (5) позволяет записать следуюшее соотношение между оператором эволюции $U(t)$ и преобразованием $\mathcal{S}(t)$ :

$$
U(t)=\mathcal{S}(t) \exp \left(-\frac{i}{\hbar} \bar{H} t\right) \mathcal{S}^{\dagger}(0) .
$$


Используя преобразование $\mathcal{S}(t)$ в уравнении (4) при $t=0$, легко установить связь между не зависящим от времени гамильтонианом $\bar{H}$ и гамильтонианом $H(t)$ в начальный момент времени $H_{0} \equiv H(0)$ :

$$
\bar{H}=\mathcal{S}^{\dagger}(0) H_{0} \mathcal{S}(0)-i \hbar \mathcal{S}^{\dagger}(0) \dot{\mathcal{S}}(0)
$$

Учет соотношения (5) дает связь между гамильтонианом в начальный и произвольный моменты времени

$$
H(t)=\mathcal{S}(t) \mathcal{S}^{\dagger}(0) H_{0} \mathcal{S}(0) \mathcal{S}^{\dagger}(t)
$$

Итак, использование унитарных преобразований, ведущих к не зависящему от времени гамильтониану, сводит решение уравнения (1) к решению уравнения (10) относительно $|\varphi(\mathcal{E})\rangle$ и решению уравнения $(7)$ относительно $\mathcal{S}(t)$. Решение квантовых задач рассеяния традиционно связано с решением одноканальных или многоканальных систем уравнений (10), описывающих стационарные процессы. Однако решение уравнения (7) относительно $\mathcal{S}(t)$ при заданном $H(t)$ в обшем случае является едва ли не более сложной задачей, чем решение исходного уравнения (1).

Мы предлагаем поступить иначе [3] - использовать в качестве исходных широкий класс не зависяших от времени гамильтонианов, для которых решения стационарного уравнения Шредингера (10) могут быть получены аналитически. Тогда при заранее заданных в определенном виде операторах $\mathcal{S}(t)$ можно найти соответствующие зависяшие от времени гамильтонианы и найти точные решения нестационарного уравнения Шредингера (1).

\section{2. ГАМИЛЬТОНИАНЫ, ДОПУСКАЮШИЕ ТОЧНЫЕ РЕШЕНИЯ НЕСТАЦИОНАРНОГО УРАВНЕНИЯ ШРЕДИНГЕРА}

Рассмотрим задачу по восстановлению в явном виде изменяющегося со временем гамильтониана

$$
H(t)=\left(\hat{p}_{x}^{2}+q(x)\right) \hat{I}+\mathbf{B}(t, x) \cdot \mathbf{j}
$$

используя не зависящие от времени гамильтонианы, допускающие аналитические решения стационарного уравнения (10). Здесь $q(x)$ - стационарный потенциал, $\mathbf{B}(t, x)$ - изменяющийся со временем потенциал, который часто можно трактовать как магнитное поле, $\mathbf{j}=\left(\hat{j}_{1}, \hat{j}_{2}, \hat{j}_{3}\right)$ - оператор спина и $\hat{j}_{i}$ - соответствуюшие ему $(2 j+1) \times$ $(2 j+1)$-матричные компоненты, $\hat{p}_{x}=-i \hbar \nabla_{x}$ - оператор импульса, $\hat{I}$ - единичная матрица.

Рассмотрим в качестве модельной стационарной задачи двухканальное уравнение Шредингера с действительной и симметричной потенциальной матрицей $V(x)\left(V_{12}(x)=\right.$ $\left.V_{21}(x)\right)$ :

$$
\bar{H}(x)|\Phi(x)\rangle=\mathcal{E}|\Phi(x)\rangle, \quad \bar{H}(x)=\hat{p}_{x}^{2}+V(x) .
$$


Как хорошо известно, по спектральным характеристикам в обратной задаче рассеяния определяют потенциал и соответствуюшие ему решения. В качестве спектральных данных служат матрица рассеяния $\mathcal{S}(k)$ и характеристики связанных состояний $\left\{\mathcal{E}_{\nu}, \gamma_{\nu}^{2}\right\}$ значения их энергий и нормировок. Гамильтониан (15) может быть представлен как сумма диагональной и бесследовой матриц

$$
\begin{aligned}
\bar{H}(x)= & \left(\hat{p}_{x}^{2}+\frac{V_{11}(x)+V_{22}(x)}{2}\right) \hat{I}+ \\
& +\left(\begin{array}{cc}
\frac{1}{2}\left(V_{11}(x)-V_{22}(x)\right) & V_{12}(x) \\
V_{21}(x) & -\frac{1}{2}\left(V_{11}(x)-V_{22}(x)\right)
\end{array}\right),
\end{aligned}
$$

что позволяет представить его в виде

$$
\bar{H}(x)=h(x) \hat{I}+\overline{\mathbf{B}}(x) \cdot \boldsymbol{\sigma}=h(x)\left(\begin{array}{ll}
1 & 0 \\
0 & 1
\end{array}\right)+\bar{\Omega}(x)\left(\begin{array}{cc}
\cos \bar{\theta}(x) & \sin \bar{\theta}(x) \\
\sin \bar{\theta}(x) & -\cos \bar{\theta}(x)
\end{array}\right) .
$$

Здесь

$$
h(x)=\hat{p}_{x}^{2}+\frac{1}{2}\left(V_{11}(x)+V_{22}(x)\right), \quad \sigma=\left(\hat{\sigma}_{1}, \hat{\sigma}_{2}, \hat{\sigma}_{3}\right),
$$

$\hat{\sigma}_{i}$ - матрицы Паули,

$$
\begin{aligned}
\overline{\mathbf{B}}(x) & =\bar{\Omega}(x)(\sin \bar{\theta}(x), 0, \cos \bar{\theta}(x)), \\
\bar{\Omega}(x) & =\frac{1}{2} \sqrt{\left(V_{11}(x)-V_{22}(x)\right)^{2}+4 V_{12}(x)^{2}}, \\
\sin \bar{\theta}(x) & =\frac{V_{12}(x)}{\bar{\Omega}(x)}, \quad \cos \bar{\theta}(x)=\frac{V_{11}(x)-V_{22}(x)}{2 \bar{\Omega}(x)} .
\end{aligned}
$$

Очевидно, что гамильтониан двухканальной задачи соответствует трехмерной задаче с координатами, зависяшими от параметра $x$. В качестве этих координат можно выбрать, например, коллективные координаты $B_{i}(x)$, зависящие от внутренних координат $x$ (в данном случае компонента $\bar{B}_{2}(x)=0$ ). Такой гамильтониан может отвечать проблеме дву хуровневого атома или движению частицы спина $1 / 2$ в переменном магнитном поле $\overline{\mathbf{B}}(x)$.

Осушествим унитарное преобразование стационарного уравнения Шредингера, используя изменяюшийся со временем оператор

$$
\mathcal{S}(t)=\exp \left(-i \hat{\sigma}_{3} \omega t\right)
$$

который соответствует врашению на угол $\omega t$ вокруг оси $z$. Из уравнения (4) получим соотношение связи между не зависящим от времени гамильтонианом $\bar{H}(x)$ и гамильтонианом $H(t)$, изменяюшимся со временем,

$$
H(t)=\mathcal{S}(t) \bar{H}(x) \mathcal{S}^{\dagger}(t)+\omega \hat{\sigma}_{3} .
$$


Гамильтониан $H(t)$ может быть переписан в виде

$$
\begin{aligned}
H(t) & =h(x)\left(\begin{array}{ll}
1 & 0 \\
0 & 1
\end{array}\right)+\bar{\Omega}(x)\left(\begin{array}{cc}
\cos \bar{\theta}(x)+\frac{\omega}{\bar{\Omega}(x)} & \sin \bar{\theta}(x) \exp (2 i \omega t) \\
\sin \bar{\theta}(x) \exp (-2 i \omega t) & -\left(\cos \bar{\theta}(x)+\frac{\omega}{\bar{\Omega}(x)}\right)
\end{array}\right)= \\
& =h(x) \hat{I}+\mathbf{B}(t, x) \cdot \boldsymbol{\sigma} .
\end{aligned}
$$

Ему можно поставить в соответствие гамильтониан (14) с нестационарным полем

$$
\mathbf{B}(t, x)=\Omega(x)(\sin \theta(x) \cos (2 \omega t), \sin \theta(x) \sin (2 \omega t), \cos \theta(x))
$$

компоненты которого определяются с помошью соотношений

$$
\begin{gathered}
\Omega(x)=\bar{\Omega}(x)\left(1+\frac{\omega^{2}}{\bar{\Omega}^{2}(x)}+2 \frac{\omega}{\bar{\Omega}(x)} \cos \bar{\theta}(x)\right)^{1 / 2} \\
\sin \theta(x)=\frac{\bar{\Omega}(x) \sin \bar{\theta}(x)}{\Omega(x)}, \quad \cos \theta(x)=\frac{\bar{\Omega}(x) \cos \bar{\theta}(x)}{\Omega(x)}+\frac{\omega}{\Omega(x)}
\end{gathered}
$$

Легко видеть из соотношений (21) и (22), что в результате унитарного преобразования (19) возникла отличная от нуля составляющая вектора $\mathbf{B}(t, x)$ вдоль оси $y$. Из соотношения (20) при $t=0$ получаем выражение для начального гамильтониана

$$
H_{0}(x)=\bar{H}(x)+\omega \hat{\sigma}_{3},
$$

которое удобно переписать в виде

$$
H_{0}(x)=\left(\hat{p}_{x}^{2}+q(x)\right) \hat{I}+\mathbf{B}_{0}(x) \cdot \boldsymbol{\sigma}
$$

где поле $\mathbf{B}_{0}$ определяется следуюшим образом:

$$
\mathbf{B}_{0}(x)=\Omega(x)(\sin \theta(x), 0, \cos \theta(x))
$$

Итак, построен гамильтониан

$$
\begin{aligned}
H(t)= & \left(\hat{p}_{x}^{2}+\frac{V_{11}(x)+V_{22}(x)}{2}\right) \hat{I}+ \\
& +\left(\begin{array}{cc}
\frac{V_{11}(x)-V_{22}(x)}{2}+\omega & V_{12}(x) \exp (2 i \omega t) \\
V_{21}(x) \exp (-2 i \omega t) & -\left(\frac{V_{11}(x)-V_{22}(x)}{2}+\omega\right)
\end{array}\right),
\end{aligned}
$$

который допускает представление в явном виде решений нестационарного уравнения Шредингера (1):

$$
|\Psi(t, x)\rangle=\exp \left(-i \hat{\sigma}_{3} \omega t\right) \exp \left(-\frac{i \bar{H} t}{\hbar}\right)|\Phi(x)\rangle .
$$


Действительно, собственные значения $\mathcal{E}$ стационарного уравнения Шредингера с гамильтонианом $\bar{H}$ известны, поскольку они являются исходными данными в обратной задаче. Используя процедуру алгебраических преобразований Баргмана или Дарбу, по спектральным характеристикам в явном виде восстанавливаются потенциал и решения $|\Phi\rangle$.

Пусть $V(x)$ - баргмановская потенциальная матрица, позволяюшая находить в явном аналитическом виде решение уравнения (15). В качестве примера приведем явный вид для матричных элементов безотражательного потенциала и соответствующих ему решений Йоста [4]:

$$
\begin{aligned}
V_{i j}(x)= & 2 \frac{d}{d x} \sum_{\nu, \lambda} \exp \left(-\kappa_{i}^{\nu} x\right) \gamma_{i}^{\nu} P_{\nu \lambda}^{-1}(x) \gamma_{j}^{\lambda} \exp \left(-\kappa_{j}^{\lambda} x\right), \\
F_{j j^{\prime}}^{ \pm}(k, x)= & \exp \left( \pm i k_{j} x\right) \delta_{j j^{\prime}}- \\
& -\sum_{\nu, \lambda} \gamma_{j}^{\nu} \exp \left(-\kappa_{j}^{\nu} x\right) P_{\nu \lambda}^{-1}(x) \int_{x}^{\infty} \gamma_{j^{\prime}}^{\lambda} \exp \left(-\left(\kappa_{j^{\prime}}^{\lambda} \pm i k_{j^{\prime}}\right) x^{\prime}\right) d x^{\prime},
\end{aligned}
$$

где

$$
P_{\nu \lambda}=\delta_{\nu \lambda}+\sum_{j^{\prime}}^{m} \frac{\gamma_{j^{\prime}}^{\nu} \gamma_{j^{\prime}}^{\lambda}}{\kappa_{j^{\prime}}^{\nu}+\kappa_{j^{\prime}}^{\lambda}} \exp \left(-\left(\kappa_{j^{\prime}}^{\nu}+\kappa_{j^{\prime}}^{\lambda}\right) x\right)
$$

Нормированное решение для векторной функции связанного состояния выражается через матрицу решений Йоста (29), взятую при энергии связанного состояния $k_{\nu}^{2}=-\mathcal{E}_{\nu}$, и имеет вид

$$
\left|\varphi_{i}\left(\mathcal{E}_{\nu}, x\right)\right\rangle=\sum_{j}^{m} F_{i j}\left(\mathcal{E}_{\nu}, x\right) \gamma_{j}^{\nu}, \quad i, j=1,2 .
$$

В приведенных формулах индексы $\nu$ и $\lambda$ отвечают связанным состояниям, характеризующимся энергиями $\mathcal{E}_{\nu}$ и нормировочными матрицами $\left|\gamma_{i}^{\nu}\right\rangle\left\langle\gamma_{j}^{\nu}\right|$, в то время как для обозначения каналовых индексов использованы индексы $i, j$. Следует отметить, что класс точно решаемых многоканальных задач далеко не ограничивается приведенным примером. Использование техники вырожденных ядер при решении интегральных уравнений обратной задачи рассеяния как на всей оси, так и на полуоси, а также радиальной задачи в подходах Гельфанда-Левитана и Марченко дает многочисленные примеры точно решаемых стационарных задач, которые могут быть использованы для исследования задач нестационарных.

\section{3. НЕАДИАБАТИЧЕСКИЕ ГЕОМЕТРИЧЕСКИЕ ФАЗЫ}

Осуществим процедуру унитарных преобразований $\overline{\mathcal{S}}(x)$ и $\mathcal{S}(x)$, устраняющих составляющие $\bar{B}_{1}(x)$ и $B_{1}(x)$ полей $\overline{\mathbf{B}}(x)$ и $\mathbf{B}_{0}(x)$. Она реализуется с помощью $S U(2)$-операторов

$$
\overline{\mathcal{S}}(x)=\exp \left(-i \bar{\theta}(x) \hat{j}_{2}\right) \quad \text { и } \mathcal{S}(x)=\exp \left(-i \theta(x) \hat{j}_{2}\right)
$$


соответственно, $\mathbf{j}=(1 / 2) \boldsymbol{\sigma}$. Поскольку справедливы соотношения

$$
\begin{aligned}
\overline{\mathbf{B}}(x) \cdot \boldsymbol{\sigma} & =\bar{\Omega}(x) \exp \left(-i \bar{\theta}(x) \hat{j}_{2}\right) \hat{\sigma}_{3} \exp \left(i \bar{\theta}(x) \hat{j}_{2}\right), \\
\mathbf{B}_{0}(x) \cdot \boldsymbol{\sigma} & =\Omega(x) \exp \left(-i \theta(x) \hat{j}_{2}\right) \hat{\sigma}_{3} \exp \left(i \theta(x) \hat{j}_{2}\right),
\end{aligned}
$$

то в результате действия преобразований $\overline{\mathcal{S}}(x)$ и $\mathcal{S}(x)$ системы уравнений для гамильтонианов (15) и (24) сводятся [5] к уравнениям калибровочного вида

$$
\begin{aligned}
& {\left[-\left(\nabla_{x} \hat{I}+\bar{A}(x)\right)^{2}+q(x) \hat{I}+\bar{\Omega}(x) \cdot \hat{\sigma}_{3}-P^{2}\right] \Phi^{\prime}(x)=0,} \\
& {\left[-\left(\nabla_{x} \hat{I}+A(x)\right)^{2}+q(x) \hat{I}+\Omega(x) \cdot \hat{\sigma}_{3}-P^{2}\right] \Psi_{0}^{\prime}(x)=0,}
\end{aligned}
$$

где $P=\operatorname{diag}\left(p_{n}\right)$, для новых функций

$$
\Phi^{\prime}(x)=\exp \left(-i \bar{\theta}(x) \hat{j}_{2}\right) \Phi(x), \quad \Psi_{0}^{\prime}(x)=\exp \left(-i \theta(x) \hat{j}_{2}\right) \Psi_{0}(x) .
$$

Связь между каналами в уравнениях $(33),(34)$ осушествляется матричными элементами операторов $\bar{A}(x)$ и $A(x)$, которые действуют как эффективные векторные потенциалы и генерируются процедурой калибровочного преобразования (30):

$$
\begin{aligned}
& \bar{A}(x)=\exp \left(i \bar{\theta}(x) \hat{j}_{2}\right) \frac{d}{d x} \exp \left(-i \bar{\theta}(x) \hat{j}_{2}\right), \\
& A(x)=\exp \left(i \theta(x) \hat{j}_{2}\right) \frac{d}{d x} \exp \left(-i \theta(x) \hat{j}_{2}\right) .
\end{aligned}
$$

Отметим, что элементы оператора

$$
\overline{\mathcal{S}}(x)=\exp \left(-i \bar{\theta}(x) \hat{j}_{2}\right)=\left(\begin{array}{cc}
\frac{\cos \bar{\theta}(x)}{2} & \frac{\sin \bar{\theta}(x)}{2} \\
-\frac{\sin \bar{\theta}(x)}{2} & \frac{\cos \bar{\theta}(x)}{2}
\end{array}\right)
$$

определяются элементами известной потенциальной матрицы $V_{i j}(x)$ по формулам $(18)$. Учитьвая оператор преобразования (19) в операторе эволюции (11):

$$
U(t)=\exp \left(-i \hat{\sigma}_{3} \omega t\right) \exp \left(-\frac{i \bar{H} t}{\hbar}\right)
$$

представим решение (27) уравнения (1) с гамильтонианом (21) в виде

$$
\left|\Psi\left(t, \mathcal{E}_{\nu}\right)\right\rangle=\exp (\mp i \omega t) \exp \left(-\frac{i \mathcal{E}_{\nu} t}{\hbar}\right)\left|\Psi\left(0, \mathcal{E}_{\nu}\right)\right\rangle
$$

Собственные значения и собственные функции оператора $\bar{H}(x)-$ это $\mathcal{E}_{\nu}$ и $\left|\varphi_{\alpha}\left(\mathcal{E}_{\nu}\right)\right\rangle$, где индекс канала $\alpha=1,2$ принимает два значения в соответствии с постановкой задачи. В момент времени $T=\pi / \omega$ имеем

$$
\left|\Psi_{\alpha}\left(T, \mathcal{E}_{\nu}\right)\right\rangle=\exp (\mp i \pi) \exp \left(-\frac{i \mathcal{E}_{\nu} T}{\hbar}\right)\left|\Psi_{\alpha}\left(0, \mathcal{E}_{\nu}\right)\right\rangle .
$$


Тогда полная фаза определится соотношением

$$
\delta_{\nu}= \pm \pi+\frac{\mathcal{E}_{\nu} T}{\hbar}
$$

Чтобы найти динамическую фазу, вычислим среднее значение $H(t)$, используя соотношения (20) и (27). Получим, что

$$
\begin{aligned}
& \left\langle\Psi_{\alpha}\left(t, \mathcal{E}_{\nu}\right)|H(t)| \Psi_{\alpha}\left(t, \mathcal{E}_{\nu}\right)\right\rangle=\left\langle\varphi_{\alpha}\left(\mathcal{E}_{\nu}\right) \mathcal{S}^{\dagger}(t)|H(t)| \mathcal{S}(t) \varphi_{\alpha}\left(\mathcal{E}_{\nu}\right)\right\rangle= \\
& \quad=\left\langle\varphi_{\alpha}\left(\mathcal{E}_{\nu}\right)|\bar{H}(x)| \varphi_{\alpha}\left(\mathcal{E}_{\nu}\right)\right\rangle+\left\langle\varphi_{\alpha}\left(\mathcal{E}_{\nu}\right)\left|\omega \hat{\sigma}_{3}\right| \varphi_{\alpha}\left(\mathcal{E}_{\nu}\right)\right\rangle=\mathcal{E}_{\nu}+\omega \overline{\hat{\sigma}}_{3}^{\nu} .
\end{aligned}
$$

С использованием выражений (35) для собственных функций $\bar{H}(x)$ среднее значение проекции спина перепишется следуюшим образом:

$$
\begin{aligned}
\overline{\hat{\sigma}}_{3}^{\nu} & =\left\langle\Psi_{\alpha}\left(t, \mathcal{E}_{\nu}\right)\left|\hat{\sigma}_{3}\right| \Psi_{\alpha}\left(t, \mathcal{E}_{\nu}\right)\right\rangle=\left\langle\varphi_{\alpha}\left(\mathcal{E}_{\nu}\right)\left|\hat{\sigma}_{3}\right| \varphi_{\alpha}\left(\mathcal{E}_{\nu}\right)\right\rangle= \\
& =\left\langle\varphi_{\alpha}^{\prime}\left(\mathcal{E}_{\nu}\right)\left|\exp \left(i \bar{\theta}(x) \hat{j}_{2}\right) \hat{\sigma}_{3} \exp \left(-i \bar{\theta}(x) \hat{j}_{2}\right)\right| \varphi_{\alpha}^{\prime}\left(\mathcal{E}_{\nu}\right)\right\rangle= \\
& =\left\langle\varphi_{\alpha}^{\prime}\left(\mathcal{E}_{\nu}\right)\left|-\sin (\bar{\theta}(x)) \hat{\sigma}_{1}+\cos (\bar{\theta}(x)) \hat{\sigma}_{3}\right| \varphi_{\alpha}^{\prime}\left(\mathcal{E}_{\nu}\right)\right\rangle .
\end{aligned}
$$

Тогда для динамической фазы $\delta_{\nu}^{\mathrm{d}}$ получим выражение

$$
\begin{aligned}
\delta_{\nu}^{\mathrm{d}} & =\int_{0}^{T}\left\langle\Psi_{\alpha}(t)|H(t)| \Psi_{\alpha}(t)\right\rangle_{\nu} d t= \\
& \left.=\frac{\mathcal{E}_{\nu} T}{\hbar}+\pi\left\langle\varphi_{\alpha}^{\prime}\left(\mathcal{E}_{\nu}\right)\right|-\sin (\bar{\theta}(x)) \hat{\sigma}_{1}+\cos (\bar{\theta}(x)) \hat{\sigma}_{3}\right)\left|\varphi_{\alpha}^{\prime}\left(\mathcal{E}_{\nu}\right)\right\rangle
\end{aligned}
$$

Учитывая (38) и (41), неадиабатическая геометрическая фаза $\delta_{\nu}^{\mathrm{g}}$ в нашем случае должна определяться из соотношения

$$
\begin{aligned}
\delta_{\nu}^{\mathrm{g}}= & \left(\delta_{\nu}-\delta_{\nu}^{\mathrm{d}}\right)=\pi\left\langle\varphi_{\alpha}^{\prime}\left(\mathcal{E}_{\nu}\right)\right| \sin (\bar{\theta}(x)) \hat{\sigma}_{1}+ \\
& +\left[ \pm 1-\cos (\bar{\theta}(x)) \hat{\sigma}_{3}\right]\left|\varphi_{\alpha}^{\prime}\left(\mathcal{E}_{\nu}\right)\right\rangle=\pi\left( \pm 1-\overline{\hat{\sigma}}_{3}^{\nu}\right) .
\end{aligned}
$$

По сути дела, геометрическая фаза задается средним значением проекции спина, полученным из (40). Это соответствует неадиабатической геометрической фазе Агаронова-Анандана [6]

$$
\delta^{\mathrm{g}}=\int_{0}^{T}\left\langle\Psi(t) \mid \frac{d}{d t} \Psi(t)\right\rangle=\int_{0}^{T}\left\langle\Phi_{\alpha}(x)\left|\mathcal{S}^{\dagger}(t) \dot{\mathcal{S}}(t)\right| \Phi_{\alpha}(x)\right\rangle=\pi \overline{\hat{\sigma}}_{3} .
$$

Совершенно очевидно, что при отсутствии зависимости от $x$ задача упрошается и переходит в задачу для частищы спина $1 / 2$ в изменяющемся со временем, но однородном в пространстве магнитном поле [7]. В адиабатическом пределе, когда $\omega / \Omega \rightarrow 0$, как видно из формул $(23), \Omega \rightarrow \bar{\Omega}, \cos \theta \rightarrow \cos \bar{\theta}$ и геометрическая фаза (42) становится фазой Берри [8]

$$
\delta^{\mathrm{g}}=\pi(1-\cos \theta)
$$

4 Теоретическая и математическая физика, т. 115, № 3, 1998 г. 
Итак, мы детально рассмотрели двухканальную точно решаемую нестационарную задачу, используя достаточно простой оператор канонического калибровочного преобразования $\mathcal{S}(t)$. Ясно, как предлагаемый подход обобщается на случаи других выборов операторов $\mathcal{S}(t)$ и классов стационарных точно решаемых задач.

\section{4. ЗАКЛЮЧЕНИЕ}

Предложена процедура построения зависящих от времени гамильтонианов, допускающих точные решения нестационарного уравнения Шредингера. Подход основан на точно решаемых моделях стационарного уравнения Шредингера и на специальном выборе операторов $S(t)$ канонического калибровочного преобразования. Детально рассмотрена двухканальная задача, для которой приведено выражение в случае фазы Берри.

Авторы признательны докторам ф.-м. наук А. Жеданову и Б. Самсонову за проявленный интерес к работе.

\section{Список литературы}

[1] Shun-Jin Wang. Phys. Rev. A. 1990. V. 42. P. 5107.

[2] Lian-Ao Wu, J. Sun, Ji-Yu Zhong. Phys. Lett. A. 1993. V. 183. P. 257.

[3] A.A. Suzko, E.P. Velicheva. Exact Solutions of Nonstationary Schrödinger Equations and Geometric Phase. In: Proc. Int. Conf. on Symmetry Methods in Physics, Dubna, July 1997; Препринт ОИЯИ Р4-97-311. Дубна: ОИЯИ, 1997.

[4] B. N. Zakhariev, A.A. Suzko. Direct and inverse problems. In: Potentials in quantum scattering. 2-nd ed. Berlin, Heidelberg, New York: Springer-Verlag, 1990.

[5] А.А. Сузько, Е. П. Величева. ЭЧАЯ. 1996. Т. 27. С. 924.

[6] Y. Aharonov, J. Anandan. J. Phys. Rev. Lett. 1987. V. 58. P. 1593.

[7] M. Stone. Phys. Rev. D. 1987. V. 33. P. 1191.

[8] M. Berry. Proc. R. Soc. London. A. 1984. V. 392. P. 45.

Поступила в редакцию 14. I.1998 г. 